\title{
Contrast Enhancing Pattern on Pre-Treatment MRI predicts Response to Anti-Angiogenic Treatment in Recurrent Glioblastoma: Comparison of Bevacizumab and Temozolomide Treatment
}

Hye Hyeon Moon

Asan Medical Center

Ji Eun Park ( $\boldsymbol{D}$ jieunp@gmail.com )

Asan Medical Center https://orcid.org/0000-0002-4419-4682

Young-Hoon Kim

Asan Medical Center

Jeong Hoon Kim

Asan Medical Center

Ho Sung Kim

Asan Medical Center

\section{Research Article}

Keywords: bevacizumab, glioblastoma, MRI, imaging biomarker, contrast enhancement

Posted Date: January 10th, 2022

DOI: https://doi.org/10.21203/rs.3.rs-1233296/v1

License: (c) (i) This work is licensed under a Creative Commons Attribution 4.0 International License.

Read Full License

Version of Record: A version of this preprint was published at Journal of Neuro-Oncology on March 11th, 2022. See the published version at https://doi.org/10.1007/s11060-022-03980-2. 


\section{Abstract}

Objective: To evaluate the value of the contrast enhancing pattern on pre-treatment MRI for predicting the response to anti-angiogenic treatment in patients with IDH-wild type recurrent glioblastoma.

Methods: This retrospective study enrolled 65 patients with IDH wild-type recurrent glioblastoma who received standard therapy and then received either bevacizumab (46 patients) or temozolomide (19 patients) as a secondary treatment. The contrast enhancing pattern on pre-treatment MRI was visually analyzed and dichotomized into contrast enhancing lesion (CEL) dominant and non-enhancing lesion (NEL) dominant types. Quantitative volumetric analysis was used to support the dichotomization. The Kaplan-Meier method and Cox proportional hazards regression analysis were used to stratify progression free survival (PFS) according to the treatment in the entire patients, CEL dominant group, and NEL dominant group.

Results: In all patients, the PFS of those treated with bevacizumab was not significantly different from those treated with temozolomide (log-rank test, $P=.96$ ). When the contrast enhancing pattern was considered, bevacizumab was associated with longer PFS in the CEL dominant group $(P=.031)$, whereas temozolomide showed longer PFS in the NEL dominant group $(P=.022)$. Quantitative analysis revealed cut-offs for the proportion of solid-enhancing tumor of $13.7 \%$ for the CEL dominant group and $4.3 \%$ for the NEL dominant group.

Conclusions: Patients with the CEL dominant type showed a better treatment response to bevacizumab, whereas NEL dominant types showed a better response to temozolomide. The contrast enhancing pattern on pre-treatment MRI can be used to stratify patients with IDH wild-type recurrent glioblastoma according to the effect of anti-angiogenic treatment.

\section{Introduction}

Anti-angiogenic treatment targeting vascular endothelial growth factor (VEGF) signaling represents a mainstay in the treatment of recurrent glioblastoma [1]. Although bevacizumab is increasingly being used in recurrent glioblastoma, tumor responses vary substantially between patients, with a lack of effective imaging biomarkers for predicting treatment response and selecting patients [2]. A predictive imaging biomarker for bevacizumab is defined by that there is a clear benefit of the bevacizumab in biomarker subgroup (positive) but a clear lack of benefit in the other biomarker subgroup (negative) [3,4]. This biomarker would be particularly useful for selecting those patients with recurrent glioblastoma who are likely to benefit from treatment with bevacizumab.

Previous imaging biomarkers used in studies on patients treated with anti-angiogenic therapy include low pre-/post-treatment relative cerebral blood volume (rCBV), little change in rCBV during treatment, a low pre-treatment $K^{\text {trans }}$ value, and a low pre-treatment apparent diffusion coefficient (ADC) value [5-9]. However, because of the single-arm nature of the studies, which did not include a control treatment group, these imaging biomarkers were prognostic rather than predictive $[3,4]$. Furthermore, they are based on 
advanced MRI techniques, and the diversity of imaging acquisition protocols and post-processing techniques used between institutions may impede the replication of single-center study results [10].

The contrast enhancing portion and peritumoral non-enhancing portion on contrast-enhanced T1weighted imaging and T2-weighted fluid-attenuated inversion recovery (FLAIR) imaging are used to define radiologic phenotypes of progression in recurrent glioblastoma [11-14]. These radiologic phenotypes are influenced by treatment, and are closely related to clinical outcomes. Nonetheless, whether the pretreatment contrast enhancement pattern on structural MRI can serve as a predictive imaging biomarker has not been studied. Previous radio-pathologic correlation studies demonstrated that protein expression patterns differ between enhancing and non-enhancing portions in glioblastoma, with enhancing portions containing more protein species in the lower molecular weight range, which may indicate that enhancing lesion is the site of increased proteolysis with destruction of the basement membrane and an increase in vascular permeability associated with angiogenesis $[15,16]$. A DNA microarray study revealed that the contrast enhancing portion tends to overexpress genes associated with the hypoxia-angiogenesis-edema pathway (such as that involving VEGF) compared with tumor showing incomplete contrast enhancement [17]. Therefore, we speculate that patients with contrast enhancing dominant tumor will respond better to anti-angiogenic treatment than patients with non-enhancing dominant tumor. In this study, we aimed to radiologically characterize isocitrate dehydrogenase (IDH)-wild type recurrent glioblastoma according to contrast enhancing and non-enhancing tumor, and investigated whether the type of radiologic predictive imaging biomarker differs between responders to treatment with bevacizumab or temozolomide.

\section{Materials And Methods}

\section{Study Population}

This retrospective data evaluation was approved by the institutional review board of Asan Medical Center (approval number:2021-1450), and the requirement for written informed consent was waived. The patients underwent secondary treatment between July 2017 and January 2021. Patients in the neurooncology databases of Asan Medical Center were retrospectively reviewed, with the inclusion criteria for recurrent glioblastoma being: (1) patients had histologically confirmed IDH wild-type glioblastoma; (2) were subsequently diagnosed with recurrence on the basis of clinical data and MRI after standard treatment of operation, concurrent chemoradiotherapy, and adjuvant temozolomide; (3) received secondary treatment of bevacizumab or temozolomide; (4) pre-treatment (before secondary treatment with bevacizumab or temozolomide) and sequential follow-up MRI including contrast-enhanced T1weighted imaging (CE-T1WI) and T2-weighted FLAIR imaging were available. Among 145 potentially eligible patients, 45 who did not have adequate pre-treatment or sequential follow-up MRI, 10 with IDH mutant type, 12 with unknown IDH status, 6 who received other secondary treatment including reoperation, re-irradiation, or other immunotherapies, and 7 with concurrent bevacizumab and temozolomide treatment were excluded. A study flowchart is presented in Figure 1. 
A total of 65 consecutive patients met the above criteria and were enrolled. All patients experienced a first recurrence after standard treatment. The included patients had: (1) regular monotherapy treatment every week with either bevacizumab (Avastin; Roche, $10 \mathrm{mg} / \mathrm{kg}$ body weight) or temozolomide (Temodal; MSD, $150 \mathrm{mg} / \mathrm{m}^{2} /$ day for 5 days every 28 days); (2) pretreatment CE-T1WI and T2-weighted FLAIR; (3) first follow-up CE-T1WI and T2-weighted FLAIR within 6 weeks after the treatment; and (4) were available for sequential follow-up MRIs.

\section{Response Assessment and Progression Free Survival}

Patients were assessed by MRI at regular 2-3 month intervals. The diagnosis of tumor progression was based on pathologic confirmation following second look operation or clinico-radiological assessment. Clinico-radiological diagnoses were made by consensus between two neuro-oncologists (J.H.K., Y.H.K; with 28 and 13 years of clinical experience in neuro-oncology, respectively) according to Response Assessment in Neuro-Oncology (RANO) criteria [18]. Radiologic progression was determined according to both enhancing and T2/FLAIR abnormalities. A quantitative increase in enhancing area (defined as a $25 \%$ or greater increase from baseline in the sum of diameter products within the contrast enhancing area), the appearance of a new lesion, or a qualitative increase in non-enhancing T2/FLAIR abnormality defined disease progression according to the RANO criteria [19].

At the time of progression, imaging patterns were determined according to whether the increased contrast enhancement or T2/FLAIR high signal intensity involved the primary site. This criterion was adopted from previous studies describing radiographic progression patterns as local, diffuse, distant, and/or multifocal progression $[11,20]$. The three main patterns of progression recorded were: 1) local enhancing progression: focus of the contrast enhancement at or within $3 \mathrm{~cm}$ of the primary site; 2) diffuse nonenhancing progression: local contrast-enhancing tumor remains stable, but an area of abnormal FLAIR hyperintensity is not concordant and extends more than $3 \mathrm{~cm}$ from the primary site; 3 ) distant progression: new focus of contrast enhancement or an area of abnormal FLAIR hyperintensity extending more than $3 \mathrm{~cm}$ from the primary site with intervening normal-appearing white matter. The judgment of progression pattern was made by two neuroradiologists in consensus.

Progression free survival (PFS) was defined as the time from secondary treatment with bevacizumab or temozolomide until the first imaging report indicating worsening/progression or death. Death was ascertained via an institutional linkage to the national healthcare system. Patients were censored at the date of medical record abstraction or the date of last imaging report, whichever came first.

\section{Imaging Protocols}

The brain tumor imaging protocol was acquired on a 3-T scanner (Ingenia 3.0 CX; Philips Healthcare, Best, the Netherlands) with a 16-channel head coil, and included the following sequences: T2-weighted, T2-weighted FLAIR, and precontrast and postcontrast T1-weighted images. T2-weighted and FLAIR images were acquired using spin echo sequences with the following parameters; T2-weighted: repetition time (TR)/echo time (TE) 3000/100 ms, field of view (FOV) $240 \times 240$ mm; matrix, $256 \times 256$; slice thickness, 4 mm without a gap; FLAIR: TR/TE 10 000/130 ms, inversion time 2800 ms, FOV $240 \times 240$ 
$\mathrm{mm}$; matrix, $256 \times 256$; and slice thickness, $4 \mathrm{~mm}$ without a gap. High-resolution anatomical threedimensional (3D) volume images were acquired using gradient-echo T1-weighted sequences with and without gadolinium-based contrast agent and with the following parameters: TR/TE 9.8/4.6 ms; flip angle, $10^{\circ}$; FOV, $256 \times 256 \mathrm{~mm}$; matrix, $512 \times 512$; and slice thickness, $1 \mathrm{~mm}$ with no gap.

\section{Determination of contrast-enhancement within T2 FLAIR high signal intensity}

Determination of dominancy type - The contrast enhancing pattern on pre-treatment MRI was classified as contrast enhancing lesion (CEL) dominant type or non-enhancing lesion (NEL) dominant type (Figure 2A). The qualitative judgment (index biomarker) of contrast enhancing pattern was independently made by two neuroradiologists (H.S.K. and J.E.P.; with 22 and 7 years of clinical experience in neuro-oncologic imaging, respectively) who were blinded to the clinical information including treatment regimen, and did not participate in any other imaging analysis.

Inter-reader agreement in the qualitative judgment (index biomarker) of contrast enhancing pattern was assessed using kappa statistics. The kappa value for interobserver agreement in the qualitative judgment (index biomarker) of contrast enhancing pattern was 0.66 (95\% confidence interval $[\mathrm{Cl}]=0.47-0.86$ ), indicating substantial agreement. Then, after a one-month wash-out period, cases with discrepancy were reread with adjudication.

Quantitative volumetric analysis using deep learning-For the quantitative analysis, The CE-T1w and FLAIR images were co-registered to define brain regions. The co-registration process was performed using Statistical Parametric Mapping (SPM12) and used affine transformations with six degrees of freedom and 4th-degree B-spline interpolation. Skull stripping was then performed with an algorithm (https://github.com/MIC-DKFZ/HD-BET). For tumour segmentation, masks of contrast-enhancing lesion (CEL), T2 FLAIR high signal intensity region (representing both edema and infiltrating tumor), and necrosis (necrotic tumor) (Figure 2B) were generated on the CE-T1 $w$ and FLAIR images using the 3D UNet-based method (https://github.com/MIC-DKFZ/nnUNet) [21] in the PyTorch package version 1.1 (Python 3.7 (www.python.org)). All image segmentations were validated by a radiologist (H.H.M, with 4 years of experience in neuroradiology). The parameter $\mathrm{Q}_{\text {whole tumor }}$ was calculated as [enhancing tumor/ (enhancing tumor + necrotic tumor + T2 FLAIR high signal intensity) $\times 100]$, while $Q_{\text {solid tumor }}$ was calculated as [enhancing tumor/ (enhancing tumor + T2 FLAIR high signal intensity) $\times 100$ ]. These parameters were used to support the determination of dominancy type.

\section{Study Design and Statistical Analysis}

The demographics and treatment responses of the patients with CEL dominant and NEL dominant types were assessed using Student's $t$-test, the chi-square test, or the Mann-Whitney test, as appropriate.

The Kaplan-Meier method was used to draw survival curves for PFS in all patients according to secondary treatment (bevacizumab or temozolomide), and the log-rank test was used to compare curves stratified by the secondary treatment. Subgroup analyses using the Kaplan-Meier method were performed 
separately in the CEL dominant and NEL dominant groups according to the secondary treatment, to investigate whether the survival outcome (PFS) differed in patients with different contrast enhancement patterns. Univariate Cox proportional hazards regression analysis was performed for all patients to evaluate whether the index imaging biomarker or secondary treatment was associated with PFS.

The two quantitative imaging parameters $\left(\mathrm{Q}_{\text {whole tumor }}\right.$ and $\mathrm{Q}_{\text {solid tumor) }}$ were compared between the CEL dominant and NEL dominant groups using the Mann-Whitney test after assessment of normality. The distribution of $\mathrm{Q}_{\text {whole tumor }}$ and $\mathrm{Q}_{\text {solid tumor }}$ in each group is displayed as a histogram.

Additionally, the effects of contrast enhancing pattern and/or secondary treatment on progression pattern were assessed by chi-square test.

All statistical analyses were performed using MedCalc version 19.2.1 or R version 3.6.3, with $P$-values $<.05$ considered statistically significant.

\section{Results}

\section{Patients}

A flow chart of the inclusion process is shown in Figure 1. Out of 65 enrolled patients, 46 (70.8\%) were treated with bevacizumab and $19(29.2 \%)$ were treated with TMZ. Qualitative judgment of contrast enhancing pattern resulted in 45 patients (69.2\%) being classified as CEL dominant and 20 patients (30.8\%) as NEL dominant. The patient demographics, treatments, and responses in the CEL dominant and NEL dominant groups are summarized in Table 1. In the CEL dominant group, there were 19 men $(42.2 \%)$ and 26 women (57.8\%). In the NEL dominant group, there were $12(60 \%)$ men and $8(40 \%)$ women. There were no significant differences in age at diagnosis, Karnofsky performance status at recurrence, or 06-methylguanine-DNA methyltransferase (MGMT) promoter methylation status between the CEL dominant and NEL dominant groups. In the CEL dominant group, 37 patients (82.2\%) were treated with bevacizumab and $8(17.8 \%)$ were treated with temozolomide. In the NEL dominant group, 9 patients (45\%) were treated with bevacizumab and 11 (55\%) were treated with temozolomide. There were no significant differences in follow-up period after treatment, diagnosis of progression up until last visit, progression free survival, or progression pattern between the CEL and NEL groups. 
Table 1

Baseline Patient Demographics and Responses

\begin{tabular}{|c|c|c|c|}
\hline & $\begin{array}{l}\text { CEL-dominant } \\
\text { Group }\end{array}$ & $\begin{array}{l}\text { NEL-dominant } \\
\text { Group }\end{array}$ & $P$ \\
\hline Clinical data & $N=45$ & $N=20$ & \\
\hline Median age (years, IQR) & $59(51-66)$ & $53(47-61.75)$ & .053 \\
\hline $\operatorname{Sex}(M / F)$ & $19 / 26$ & $12 / 8$ & .019 \\
\hline Median KPS at recurrence & $80(80-100)$ & $90(80-100)$ & .874 \\
\hline $\begin{array}{l}\text { MGMT promoter methylation status } \\
\text { (methylated/unmethylated/not available) }\end{array}$ & $18 / 16 / 11$ & $5 / 12 / 3$ & .307 \\
\hline Extent of resection at pretreatment surgery & & & .289 \\
\hline Biopsy & 4 & 1 & \\
\hline Partial/subtotal resection & 14 & 3 & \\
\hline Gross total resection & 27 & 16 & \\
\hline Adjuvant treatment & & & .002 \\
\hline Bevacizumab & 37 & 9 & \\
\hline Temozolomide & 8 & 11 & \\
\hline \multicolumn{4}{|l|}{ Response } \\
\hline Follow-up periods after adjuvant treatment (median, IQR) & $\begin{array}{l}162(77-311) \\
\text { days }\end{array}$ & $\begin{array}{l}269(87-358) \\
\text { days }\end{array}$ & .255 \\
\hline Diagnosis of progression up until last visit & $\begin{array}{l}39(86.7 \% \\
[39 / 45])\end{array}$ & $\begin{array}{l}17(85 \%, \\
[17 / 20])\end{array}$ & .859 \\
\hline Progression free survival (median, IQR) & $\begin{array}{l}97(59-135) \\
\text { days }\end{array}$ & $\begin{array}{l}98(58-207) \\
\text { days }\end{array}$ & .738 \\
\hline Progression pattern & & & .984 \\
\hline Local enhancing progression & 17 & 7 & \\
\hline Diffuse non-enhancing progression & 14 & 7 & \\
\hline Distant progression & 8 & 3 & \\
\hline
\end{tabular}

Progression Free Survival Stratified by Imaging Biomarker and Secondary Treatment 
Figure 3A demonstrates Kaplan-Meier survival curves for all patients according to secondary treatment. In the entire patients, the PFS of those treated with bevacizumab was not significantly different from those treated with temozolomide (log-rank test, $P=.96)$.

Survival outcomes were then assessed separately for the CEL dominant and NEL dominant groups (Figure 3B and C). In the CEL dominant group, the patients treated with bevacizumab showed longer PFS than those treated with temozolomide (log-rank test, $P=.031$ ). Conversely, in the NEL dominant group, the patients treated with temozolomide showed longer PFS than those treated with bevacizumab (log-rank test, $P=$.022). The univariate Cox proportional hazards regression analysis showed that bevacizumab was associated with longer PFS in the CEL dominant group (hazard ratio [HR] 0.418, 95\% Cl 0.184-.951; $P=.038$ ), but shorter PFS in the NEL dominant group (HR 3.386, 95\% $\mathrm{Cl} 1.115-10.284 ; P=.031$ ) (Table 2).

Table 2

Univariate Cox Proportional Hazard Analysis of Prediction of Progression Free Survival in Patients Stratified by Imaging Biomarker and Secondary Treatment

\begin{tabular}{|c|c|c|c|c|}
\hline & & Hazard ratio & $95 \% \mathrm{Cl}$ & $P$-value \\
\hline \multirow[t]{2}{*}{ Entire patient } & Bevacizumab & 1.082 & $0.605-1.936$ & 0.790 \\
\hline & Temozolomide & ref & & \\
\hline \multirow[t]{2}{*}{ CEL-dominant group } & Bevacizumab & 0.418 & $0.184-0.951$ & 0.038 \\
\hline & Temozolomide & ref & & \\
\hline \multirow[t]{2}{*}{ NEL-dominant group } & Bevacizumab & 3.386 & $1.115-10.284$ & 0.031 \\
\hline & Temozolomide & ref & & \\
\hline \multicolumn{5}{|c|}{ Hazard ratios reported here indicate relative change in hazard from 1 unit increases in each treatmen } \\
\hline
\end{tabular}

In the entire patients, the progression patterns differed significantly according to the secondary treatment (chi-square test, $P=.001)$. In the patients treated with bevacizumab $(\mathrm{n}=46), 40$ showed progression, of which 13 showed local enhancing progression, 20 diffuse non-enhancing progression, and 7 distant progression. In the patients treated with temozolomide $(n=19), 16$ showed progression, of which 11 showed local enhancing progression, 1 diffuse non-enhancing progression, and 4 distant progression. However, the progression patterns did not significantly differ according to the initial contrast enhancing pattern (chi-square test, $P=.926$ ).

\section{Derivation of the Imaging Biomarker with Qualitative and Quantitative Volumetric Analysis}


A histogram showing the distribution of $\mathrm{Q}_{\text {whole tumor }}$ and $\mathrm{Q}_{\text {solid tumor }}$ in the CEL dominant and NEL dominant types is shown in Supplementary Figure 1 . The average $\mathrm{Q}_{\text {whole tumor }}$ in the CEL dominant group was $13.7 \%$ of contrast enhancement in the entire tumor portion (standard deviation [SD], $9.6 \%$; range, 0.1-51.9\%), whereas in the NEL dominant group it was 4.3\% (SD 5\%; range, 0.2-18.7\%). Similarly, $Q_{\text {solid }}$ tumor was $15.1 \%$ (SD, $11.2 \%$; range, $0.1-57.8 \%$ ) in the CEL dominant group and $4.5 \%$ (SD, $5.4 \%$; range, $0.2-20.2 \%)$ in the NEL dominant group. The volumetry of the CEL group was significantly different to that of the NEL group (Mann-Whitney test, largest $P<.0001$ ).

\section{Discussion}

This study investigated the predictive value of the contrast enhancing pattern on pre-treatment MRI for stratifying patients with IDH-wild type recurrent glioblastoma according to treatment response on antiangiogenic treatment. In the CEL dominant group, bevacizumab showed a clear benefit (longer PFS) compared with temozolomide, but in the NEL dominant group, bevacizumab showed no benefit, but rather harm (shorter PFS) compared with temozolomide. These results suggest that patients with the CEL dominant type might have a better treatment response to anti-angiogenic treatment, and that the contrast enhancing pattern seems to be a promising predictive imaging biomarker for pre-treatment stratification of patients according to predicted treatment response on anti-angiogenic treatment.

Contrast enhancing tumor represents the angiogenic component of glioblastoma, which tends to be driven by VEGF [22]. Overexpression of VEGF in contrast enhancing tumor promotes angiogenesis, forming immature tumor vessels with excessive leakiness, which can contribute to disruption of the blood-brain barrier (BBB) $[23,24]$. Furthermore, contrast enhancing tumor is thought to be the site of increased proteolysis, with the presence of matrix metalloproteinases, destruction of the basement membrane, and increased vascular density and permeability [16]. Therefore, the combination of increased VEGF level and efficient drug delivery with increased vascular permeability and microvascular density in CEL dominant type glioblastoma might result in favorable treatment outcomes with anti-angiogenic treatment targeting VEGF signaling. Pope et al. [25] revealed that anti-VEGF therapy suppresses contrast enhancing tumor more effectively than non-enhancing tumor in the same patient. Liu et al. [26] described a subgroup of patients with elevated perfusion features (angiogenic subgroup) that were associated with poor overall survival, but significantly better survival when treated with anti-angiogenic treatment than not treated.

There are several potential imaging biomarkers for antiangiogenic treatment, with a low pre- or posttreatment $\mathrm{rCBV}$, low change in rCBV during treatment, low pre-treatment Ktrans, low pre-treatment ADC values for the lower peak of bimodal histogram anlalysis, and small $A D C$ volume changes during treatment all indicating a good prognosis $[6-9,27]$. However, these observations are limited because the imaging studies describing them did not include a temozolomide treatment group as a comparison, and they can only be considered as prognostic markers, rather than predictive markers of treatment. Furthermore, their predictions were based on advanced MRI techniques (including perfusion-weighted imaging and diffusion-weighted imaging) or differences in MRI features between pre- and post-treatment 
examinations. Here, we describe a potential predictive imaging biomarker using the contrast enhancing pattern on conventional MRI for the pre-treatment stratification of patients according to the predicted treatment effect of anti-angiogenic therapy in comparison with temozolomide.

Non-enhancing tumor that appears as T2 FLAIR hyperintensity represents the invasive or migratory component of glioblastoma, from which viable and proliferating tumor cells invade adjacent highly vascularized normal tissue [22]. Tumor cells in such areas can co-opt the pre-existent normal vasculature to acquire their blood supply and metabolic support [28]. These co-opted vessels are refractory to antiangiogenic treatment and offer an efficient escape mechanism against anti-angiogenic therapy [29]. Disruption of the BBB is an important factor in transendothelial diffusion of anticancer agents, because large molecules have difficulty passing the BBB if it is not fully disrupted [30,31]. Non-enhancing tumor tends to have a relatively intact BBB, and this might explain the low treatment efficacy of bevacizumab (molecular weight $=149 \mathrm{kDa})$ in comparison with temozolomide (molecular weight $=194 \mathrm{Da})$ in patients with NEL dominant type glioblastoma. Although the importance of non-enhancing tumor in glioblastoma has been emphasized in recent studies [32,33], the prognostic and predictive values of non-enhancing tumor in recurrent glioblastoma are poorly understood. Molecular imaging techniques such as amide proton transfer imaging [34] may produce helpful imaging biomarkers for NEL after antiangiogenic therapy, but further study is required to investigate their use as predictive imaging biomarkers for stratifying patients with NEL dominant type glioblastoma according to treatment response.

In this study, we performed quantitative volumetric analysis using deep learning segmentation, which segmented the enhancing tumor region, necrotic tumor region, and T2 FLAIR high signal intensity region on CE-T1WI and FLAIR images. This quantitative analysis matched with the qualitative determination (index biomarker) of the contrast enhancing pattern, which showed substantial agreement between two readers. This result suggests that the contrast enhancing pattern is a reliable and reproducible imaging biomarker that can be generally accepted in multicenter clinical practice.

Radiographic progression patterns have been categorized using standard-of-care imaging into local enhancing, diffuse non-enhancing, and distant progression types [11, 20]. Our study found the frequencies of these progression patterns varied depending on the secondary treatment. A substantial proportion of patients treated with bevacizumab showed diffuse non-enhancing progression, whereas a substantial proportion of patients treated with temozolomide showed local enhancing progression. Diffuse non-enhancing progression is promoted by prolonged blockade of vascular proliferation and tumor escape through vascular co-option, whereas local enhancing progression indicates failure of local disease control [34]. Several previous studies have shown that anti-VEGF therapy facilitates co-option of the normal vasculature and tumor invasion, consequently promoting non-enhancing diffuse tumor infiltration $[35,36]$. Our findings are consistent with a previous meta-analysis, in that high-grade glioma patients tend to show non-local or non-enhancing radiologic patterns of recurrence after bevacizumab [14], which may suggest that bevacizumab effectively controls local tumor growth, but either fails to control or actually promotes distant and diffuse recurrences. 
This study has several limitations in addition to those due to its retrospective nature. First, histological confirmation was not possible at the time of radiographic progression because of the invasiveness of the required procedures. Second, the number of patients treated with temozolomide was relatively small because bevacizumab is the major standard treatment for recurrent glioblastoma in our institution. Third, we did not incorporate the results of advanced MRI, including DWI and perfusion-weighted imaging, which increase the diagnostic accuracy of progression and help to assess treatment response. However, the lack of standardization in advanced imaging protocols has prevented their use as reproducible imaging biomarkers in multicenter practices.

In conclusion, we found that patients with CEL dominant type recurrent glioblastoma had a better treatment response to bevacizumab than those with the NEL dominant type. The contrast enhancing pattern on pre-treatment MRI can be used as a reliable and reproducible imaging biomarker to stratify patients according to treatment response on anti-angiogenic treatment, and to personalize treatment planning in patients with IDH wild-type recurrent glioblastoma.

\section{Declarations}

\section{Conflict of interest}

All the authors have nothing to disclose.

\section{Author contributions}

All authors reviewed the manuscript. H.H.M. contributed to formal analysis, visualization, and writing the original draft and editing the manuscript. J.E.P. contributed to conceptualization, data curation, formal analysis, investigation, methodology, writing and revising the manuscript. Y.H.K. contributed to data curation and formal analysis. J.H.K. contributed to data curation, project administration, and project integrity. H.S.K. contributed to conceptualization, formal analysis, investigation, supervision, editing the manuscript, and project integrity.

\section{Ethical approval}

This study was conducted retrospectively using data obtained for clinical purposes. This study was approved by the Internal Review Board of the Asan Medical Center (Approval Number: 2021-1450).

\section{Data availability}

The datasets generated and analyzed in the current study are available from the corresponding author on reasonable request.

\section{References}


1. Kreisl TN, Kim L, Moore K, Duic P, Royce C, Stroud I, Garren N, Mackey M, Butman JA, Camphausen K (2009) Phase II trial of single-agent bevacizumab followed by bevacizumab plus irinotecan at tumor progression in recurrent glioblastoma. J Clin Oncol 27:740

2. Norden AD, Drappatz J, Muzikansky A, David K, Gerard M, McNamara MB, Phan P, Ross A, Kesari S, Wen PY (2009) An exploratory survival analysis of anti-angiogenic therapy for recurrent malignant glioma. J Neurooncol 92:149-155

3. Ballman KV (2015) Biomarker: Predictive or Prognostic? 33:3968-3971.. doi: $10.1200 / j c o .2015 .63 .3651$

4. Oldenhuis CN, Oosting SF, Gietema JA, de Vries EG (2008) Prognostic versus predictive value of biomarkers in oncology. Eur J Cancer 44:946-953. doi: 10.1016/j.ejca.2008.03.006

5. Bennett IE, Field KM, Hovens CM, Moffat BA, Rosenthal MA, Drummond K, Kaye AH, Morokoff AP (2017) Early perfusion MRI predicts survival outcome in patients with recurrent glioblastoma treated with bevacizumab and carboplatin. J Neurooncol 131:321-329. doi: 10.1007/s11060-016-2300-0

6. Kickingereder P, Wiestler B, Burth S, Wick A, Nowosielski M, Heiland S, Schlemmer H-P, Wick W, Bendszus M, Radbruch A (2015) Relative cerebral blood volume is a potential predictive imaging biomarker of bevacizumab efficacy in recurrent glioblastoma. Neurooncology 17:1139-1147. doi: 10.1093/neuonc/nov028

7. Kong Z, Yan C, Zhu R, Wang J, Wang Y, Wang Y, Wang R, Feng F, Ma W (2018) Imaging biomarkers guided anti-angiogenic therapy for malignant gliomas. Neurolmage: Clinical 20:51-60

8. Pope WB, Lai A, Mehta R, Kim H, Qiao J, Young JR, Xue X, Goldin J, Brown M, Nghiemphu P (2011) Apparent diffusion coefficient histogram analysis stratifies progression-free survival in newly diagnosed bevacizumab-treated glioblastoma. American Journal of Neuroradiology 32:882-889

9. Schmainda KM, Zhang Z, Prah M, Snyder BS, Gilbert MR, Sorensen AG, Barboriak DP, Boxerman JL (2015) Dynamic susceptibility contrast MRI measures of relative cerebral blood volume as a prognostic marker for overall survival in recurrent glioblastoma: results from the ACRIN 6677/RTOG 0625 multicenter trial. Neurooncology 17:1148-1156

10. Zhang J, Liu H, Tong H, Wang S, Yang Y, Liu G, Zhang W (2017) Clinical applications of contrastenhanced perfusion MRI techniques in gliomas: recent advances and current challenges. Contrast media \& molecular imaging 2017

11. Nowosielski M, Wiestler B, Goebel G, Hutterer M, Schlemmer HP, Stockhammer G, Wick W, Bendszus $M$, Radbruch $A$ (2014) Progression types after antiangiogenic therapy are related to outcome in recurrent glioblastoma. Neurology 82:1684-1692

12. Cachia D, Elshafeey NA, Kamiya-Matsuoka C, Hatami M, Alfaro-Munoz KD, Mandel JJ, Colen R, DeGroot JF (2017) Radiographic patterns of progression with associated outcomes after bevacizumab therapy in glioblastoma patients. J Neurooncol 135:75-81. doi: 10.1007/s11060-0172550-5

13. Nowosielski M, Ellingson BM, Chinot OL, Garcia J, Revil C, Radbruch A, Nishikawa R, Mason WP, Henriksson R, Saran F (2018) Radiologic progression of glioblastoma under therapy-an exploratory 
analysis of AVAglio. Neurooncology 20:557-566

14. Cho SJ, Kim HS, Suh CH, Park JE (2020) Radiological Recurrence Patterns after Bevacizumab Treatment of Recurrent High-Grade Glioma: A Systematic Review and Meta-Analysis. Korean J Radiol 21:908-918. doi: 10.3348/kjr.2019.0898

15. Azok J, Guccione S, Lim M, Harsh G, Atlas S, Li K, Bednarski M The use of magnetic resonance imaging to guide the analysis of serum proteomic patterns in glioblastoma multiforme.Proc Intl Soc Mag Reson Med, p220

16. Hobbs SK, Shi G, Homer R, Harsh G, Atlas SW, Bednarski MD (2003) Magnetic resonance imageguided proteomics of human glioblastoma multiforme. Journal of Magnetic Resonance Imaging: An Official Journal of the International Society for Magnetic Resonance in Medicine 18:530-536

17. Pope WB, Chen JH, Dong J, Carlson MR, Perlina A, Cloughesy TF, Liau LM, Mischel PS, Nghiemphu P, Lai A (2008) Relationship between gene expression and enhancement in glioblastoma multiforme: exploratory DNA microarray analysis. Radiology 249:268-277

18. Wen PY, Macdonald DR, Reardon DA, Cloughesy TF, Sorensen AG, Galanis E, DeGroot J, Wick W, Gilbert MR, Lassman AB (2010) Updated response assessment criteria for high-grade gliomas: response assessment in neuro-oncology working group. J Clin Oncol 28:1963-1972

19. Huang RY, Rahman R, Ballman KV, Felten SJ, Anderson SK, Ellingson BM, Nayak L, Lee EQ, Abrey LE, Galanis E, Reardon DA, Pope WB, Cloughesy TF, Wen PY (2016) The Impact of T2/FLAIR Evaluation per RANO Criteria on Response Assessment of Recurrent Glioblastoma Patients Treated with Bevacizumab. Clin Cancer Res 22:575-581. doi: 10.1158/1078-0432.Ccr-14-3040

20. Pope W, Xia Q, Paton V, Das A, Hambleton J, Kim H, Huo J, Brown M, Goldin J, Cloughesy T (2011) Patterns of progression in patients with recurrent glioblastoma treated with bevacizumab. Neurology 76:432-437

21. Isensee F, Schell M, Pflueger I, Brugnara G, Bonekamp D, Neuberger U, Wick A, Schlemmer HP, Heiland S, Wick W, Bendszus M, Maier-Hein KH, Kickingereder P (2019) Automated brain extraction of multisequence MRI using artificial neural networks. J Time Ser Anal 40:4952-4964. doi: $10.1002 / \mathrm{hbm} .24750$

22. Verhoeff JJC, van Tellingen $O$, Claes A, Stalpers LJA, van Linde ME, Richel DJ, Leenders WPJ, van Furth WR (2009) Concerns about anti-angiogenic treatment in patients with glioblastoma multiforme. BMC Cancer 9:444. doi: 10.1186/1471-2407-9-444

23. Jain RK (2003) Molecular regulation of vessel maturation. Nat Med 9:685-693. doi: 10.1038/nm0603-685

24. Hardee ME, Zagzag D (2012) Mechanisms of glioma-associated neovascularization. Am J Pathol 181:1126-1141

25. Pope WB, Lai A, Nghiemphu P, Mischel P, Cloughesy T (2006) MRI in patients with high-grade gliomas treated with bevacizumab and chemotherapy. Neurology 66:1258-1260

26. Liu TT, Achrol AS, Mitchell LA, Rodriguez SA, Feroze A, Iv M, Kim C, Chaudhary N, Gevaert O, Stuart JM (2017) Magnetic resonance perfusion image features uncover an angiogenic subgroup of 
glioblastoma patients with poor survival and better response to antiangiogenic treatment. Neurooncology 19:997-1007

27. Zhang W, Kreisl T, Solomon J, Reynolds R, Glen D, Cox R, Fine H, Butman J Acute effects of bevacizumab on glioblastoma vascularity assessed with DCE-MRI and relation to patient survival. Proceedings ISMRM, p 282

28. Lamszus K, Kunkel P, Westphal M (2003) Invasion as limitation to anti-angiogenic glioma therapy. Acta Neurochir Suppl 88:169-177. doi: 10.1007/978-3-7091-6090-9_23

29. van Kempen LC, Leenders WP (2006) Tumours can adapt to anti-angiogenic therapy depending on the stromal context: lessons from endothelial cell biology. Eur J Cell Biol 85:61-68. doi: 10.1016/j.ejcb.2005.10.003

30. Mathieu V, De Nève N, Le Mercier M, Dewelle J, Gaussin J-F, Dehoux M, Kiss R, Lefranc F (2008) Combining bevacizumab with temozolomide increases the antitumor efficacy of temozolomide in a human glioblastoma orthotopic xenograft model. Neoplasia 10:1383-1392

31. Leten C, Struys T, Dresselaers T, Himmelreich U (2014) In vivo and ex vivo assessment of the blood brain barrier integrity in different glioblastoma animal models. J Neurooncol 119:297-306

32. Lasocki A, Gaillard F, Tacey M, Drummond K, Stuckey S (2016) Incidence and prognostic significance of non-enhancing cortical signal abnormality in glioblastoma. J Med Imaging Radiat Oncol 60:6673

33. Kotrotsou A, Elakkad A, Sun J, Thomas GA, Yang D, Abrol S, Wei W, Weinberg JS, Bakhtiari AS, Kircher MF (2018) Multi-center study finds postoperative residual non-enhancing component of glioblastoma as a new determinant of patient outcome. J Neurooncol 139:125-133

34. Park JE, Kim HS, Park SY, Jung SC, Kim JH, Heo H-Y (2020) Identification of Early Response to AntiAngiogenic Therapy in Recurrent Glioblastoma: Amide Proton Transfer-weighted and Perfusionweighted MRI compared with Diffusion-weighted MRI. Radiology 295:397-406

35. de Groot JF, Fuller G, Kumar AJ, Piao Y, Eterovic K, Ji Y, Conrad CA (2010) Tumor invasion after treatment of glioblastoma with bevacizumab: radiographic and pathologic correlation in humans and mice. Neuro Oncol 12:233-242. doi: 10.1093/neuonc/nop027

36. Norden AD, Young GS, Setayesh K, Muzikansky A, Klufas R, Ross GL, Ciampa AS, Ebbeling LG, Levy B, Drappatz J, Kesari S, Wen PY (2008) Bevacizumab for recurrent malignant gliomas. Neurology 70:779. doi: 10.1212/01.wnl.0000304121.57857.38

\section{Figures}




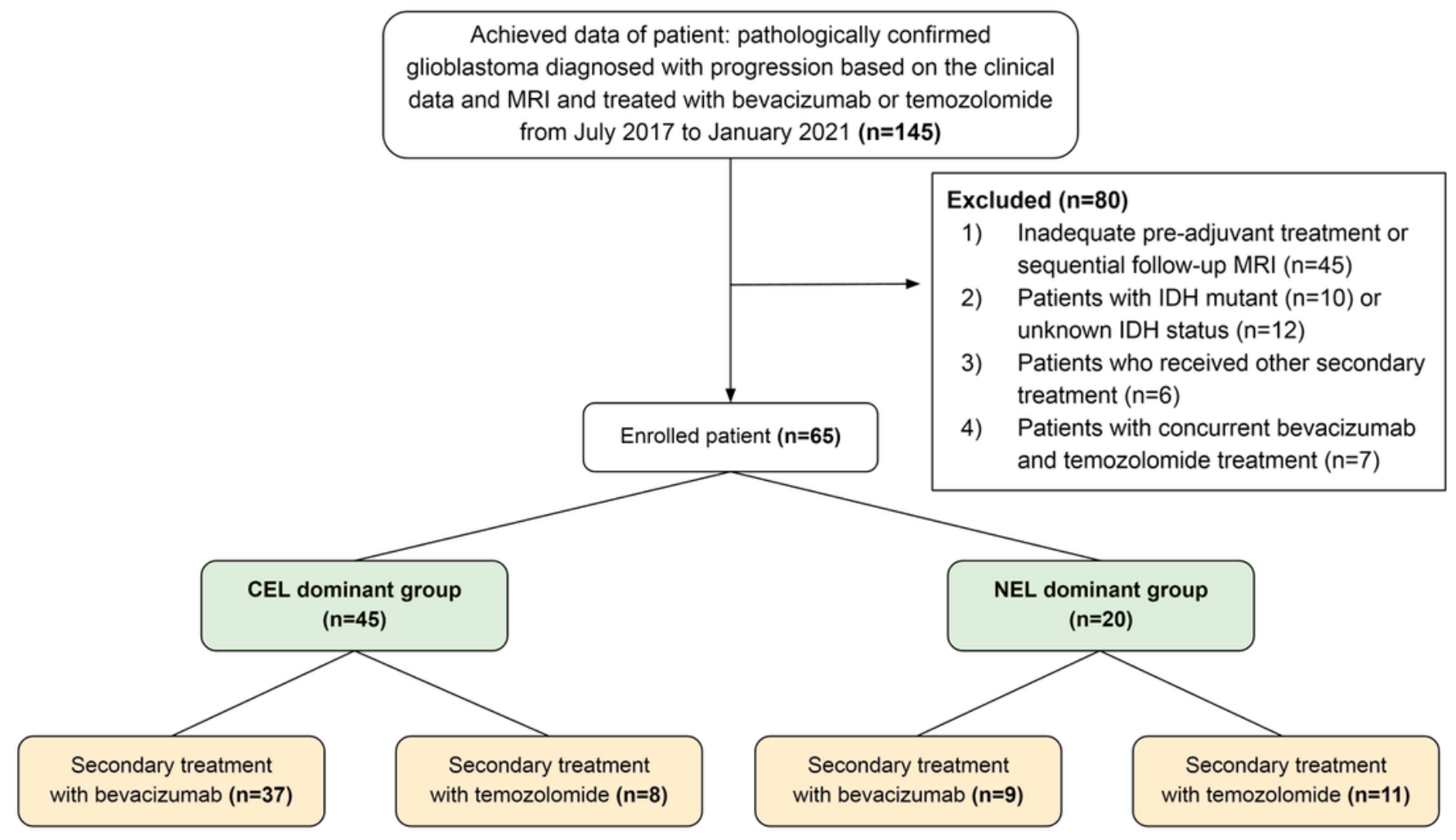

\section{Figure 1}

Flowchart showing the inclusion and exclusion process 


\section{(A)}
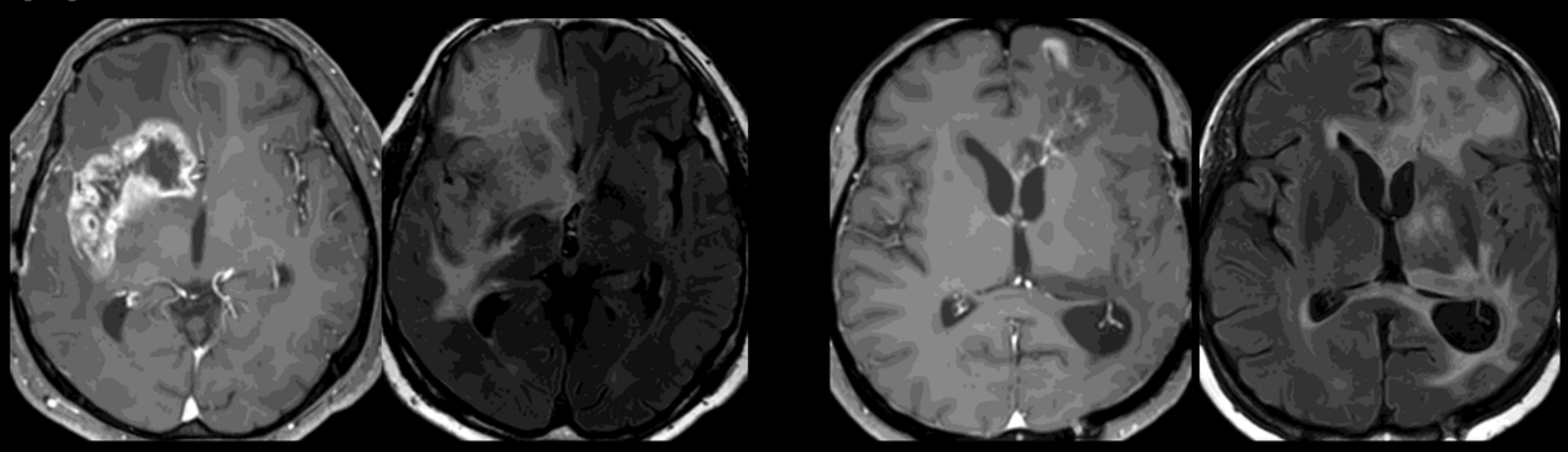

(B)
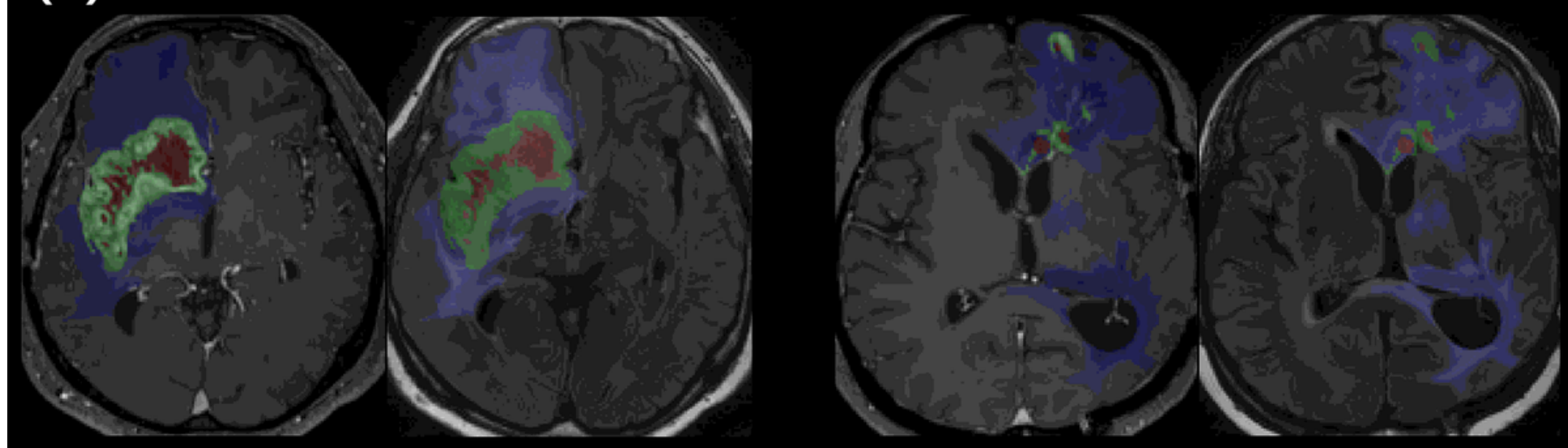

\section{Figure 2}

(A) Representative patient images for classification of contrast enhancing pattern on pre-treatment T2 FLAIR and contrast-enhanced T1-weighted images. A contrast enhancing lesion dominant type is shown on the left and a non-enhancing lesion dominant type on the right. (B) Representative patient images for quantitative volumetric analysis. Color code for segmentations: red = necrotic tumor region, green $=$ enhancing tumor region, blue $=$ T2 FLAIR high signal intensity region. Parameter values were $Q_{\text {whole tumor }}$, $15.1 \%$, and $Q_{\text {solid tumor }}, 15.6 \%$ for contrast enhancing lesion dominant type (left); and $Q_{\text {whole tumor }}, 0.1 \%$, and $\mathrm{Q}_{\text {solid tumor }}, 0.1 \%$ for non-enhancing lesion dominant type (right). 

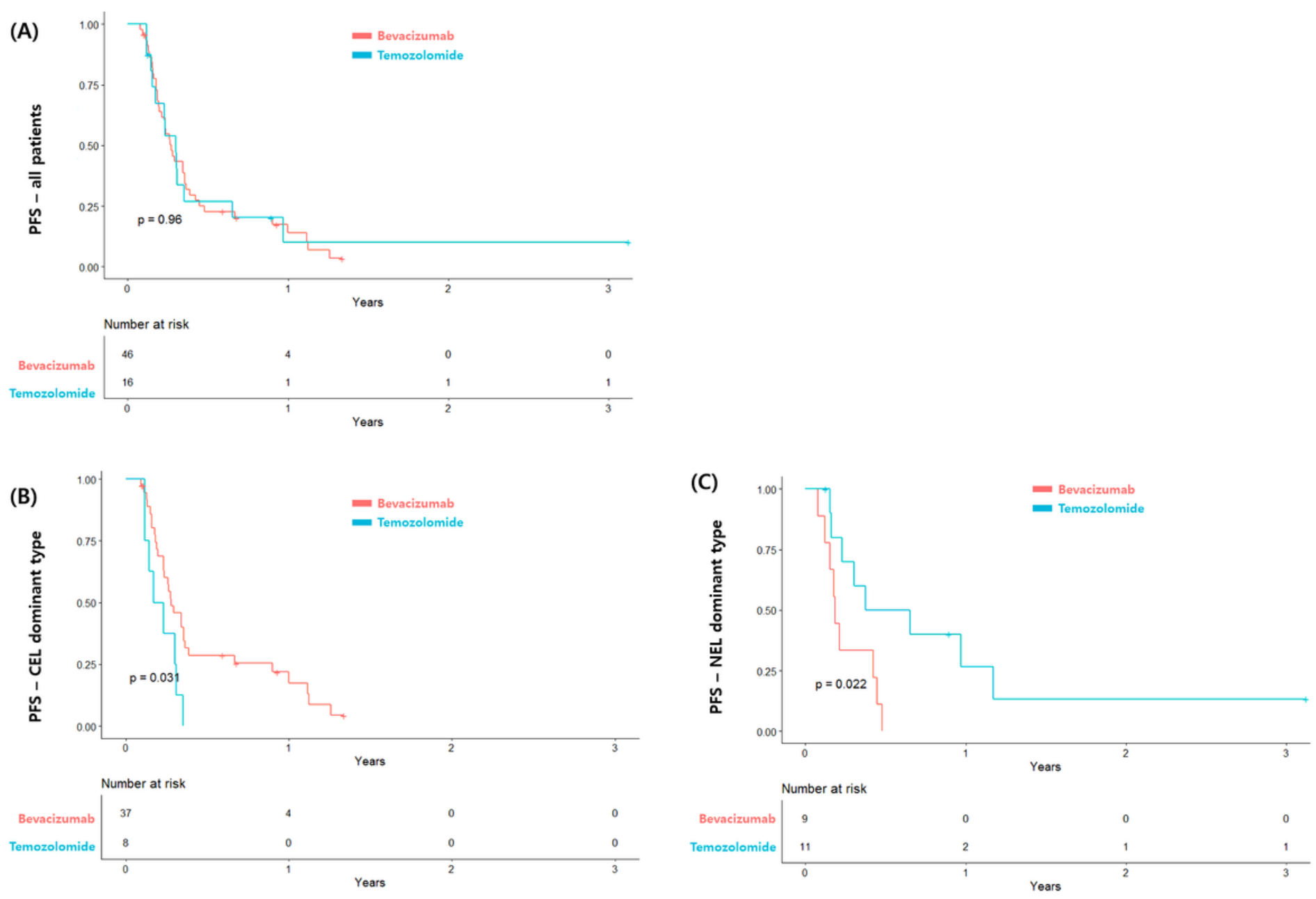

Figure 3

Kaplan-Meier survival curves for (A) bevacizumab versus temozolomide-treated patients in the entire patients $(n=65)$, and (B) CEL dominant type and (C) NEL dominant type patients. Cross-hatches represent censored data. (A) Stratification based on secondary treatment in patients with IDH wild-type glioblastoma did not result in significant differences in PFS. (B) In patients with CEL dominant type tumor, survival was better in those treated with bevacizumab $(n=37)$ than in those treated with temozolomide $(n=8)$. (C) In patients with NEL dominant type tumor, survival was better in those treated with temozolomide $(n=11)$ than in those treated with bevacizumab $(n=9)$.

\section{Supplementary Files}

This is a list of supplementary files associated with this preprint. Click to download.

- SupplementalFigure1.png 